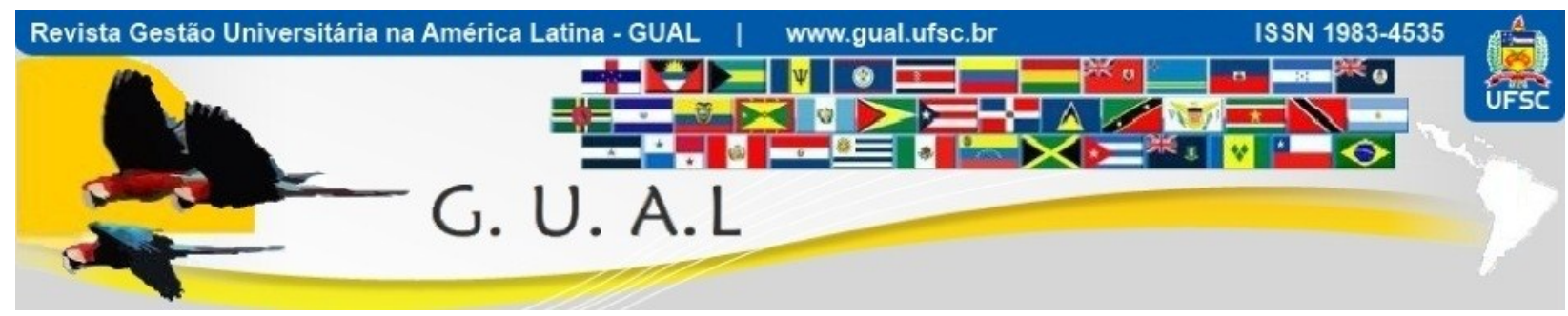

DOI: http://dx.doi.org/10.5007/1983-4535.2012v5n4p188

\title{
INVESTIGACIÓN COMPARADA EN EDUCACIÓN: PROCESOS DE INCORPORACIÓN DE LAS TIC EN LA PRÁCTICA DOCENTE DEL SUAYED-FFYL UNAM
}

\section{COMPARATIVE STUDIES IN EDUCATION: THE USE OF ICT IN THE TEACHING PRACTICE OF SUAYED, UNAM-FFYL}

Ileana Rojas Moreno, Mestre Universidad Nacional Autónoma de México - UNAM ileana.rojas.moreno@gmail.com

Zaira Navarrete Cazales, Mestre Universidad Nacional Autónoma de México - UNAM znavarretec@gmail.com

José Miguel Sánchez Alemán, Mestre Universidad Nacional Autónoma de México - UNAM josemiguel789@hotmail.com

Recebido em 16/novembro/2012

Aprovado em 07/dezembro/2012

Sistema de Avaliação: Double Blind Review

Esta obra está sob uma Licença Creative Commons Atribuição-Uso. 


\title{
RESUMEN
}

El proyecto de investigación que presentamos tiene como propósito central la preparación de un estado del conocimiento que caracterice un panorama de corte comparativo sobre la práctica docente de los profesores del Sistema de Universidad Abierta y Educación a Distancia de la Facultad de Filosofía y Letras de la UNAM, (SUAyED/FFyL-UNAM), destacando en particular los procesos de incorporación y manejo de las Tecnologías de la Información y la Comunicación (TIC) en el trabajo docente cotidiano en condiciones de no presencialidad (enseñanza abierta, enseñanza virtual), a fin de analizar aportes, ventajas y limitaciones de esta tendencia actual.

Un aspecto nodal de esta investigación consiste en ubicar, comparar y analizar las especificidades en cuanto a la incorporación y el manejo de las TIC por parte de los profesores de las diversas Licenciaturas que conforman la planta académica de la División SUAyED/FFyL. Estimamos que los resultados de este trabajo representarán un aporte de base para la toma de decisiones posteriores relacionadas con aspectos como contratación de personal, procesos de actualización, productividad y evaluación académicas, proyectos de trabajo colegiado, entre otros. Asimismo, consideramos que todo lo anterior redundará de manera definitiva en favor del rendimiento académico de los estudiantes universitarios de esta modalidad educativa.

Palabras-clave: TIC. Investigación comparada. Docencia. Evaluación.

\begin{abstract}
Our research project has as main purpose to prepare state of art, from a comparative studies vision, about teaching practice of Open University and Distance Education faculty at the Faculty of Philosophy and Language Arts of the UNAM (SUAyED/ FFyL-UNAM), highlighting the processes of incorporation and management of Information and Communication Technology (ITC) in the every day e-learning system teaching work, and analyzing contributions, advantages and limitations of this current trend. A nodal aspect of this research is to locate, compare and analyze particular details on teachers ITC incorporation and management. We believe that the results of this study represent a contribution for future decision-making related issues such as staffing, updating processes, productivity and academic assessment, academic work projects, among many others. We also believe that the result definitively will contribute to improve the academic performance of distance education college students.
\end{abstract}

Keywords: ICT. Comparative studies. Teaching. Assessment. 


\section{INVESTIGACIÓN COMPARADA EN EDUCACIÓN: PROCESOS DE INCORPORACIÓN DE LAS \\ TIC EN LA PRÁCTICA DOCENTE DEL SUAYED-FFYL UNAM \\ DOI: http://dx.doi.org/10.5007/1983-4535.2012v5n4p188}

\section{INTRODUCCIÓN}

En el contexto de la globalización como tendencia económica mundial y a partir de la llamada "tercera revolución tecnológica", el mundo ha sido testigo de cambios vertiginosos encaminados hacia la construcción de la "sociedad informatizada". Desde esta perspectiva, hablar de una cultura posmoderna, la realidad virtual, el ciberespacio, las nuevas tecnologías de la información y la comunicación (TIC), obliga a revisar algunos de los argumentos que analizan bajo diferentes lógicas esta visión compleja del actual panorama, particularmente sobre la formación, profesionalización y desempeño de docentes de nivel superior en México ante los retos que enfrentan los procesos educativos, dada la crisis de los saberes y la emergencia de nuevos conocimientos y demandas al inicio del nuevo milenio.

Con base en lo anterior, consideramos que la condición formativa y de desempeño del profesorado no puede encasillarse solamente en aspectos técnicos sin correr el riesgo de reducir la docencia a una actividad mecánica. No obstante, no podemos obviar la importancia de la incorporación y el manejo didáctico de las TIC en la práctica educativa.

Actualmente, los sistemas de educación en modalidades no presenciales (abierta, a distancia) representan un reto para la educación universitaria en general, a la vez que encierran grandes posibilidades en la formación continua de recursos humanos tan necesaria para el desarrollo personal e institucional. En el caso de esta modalidad educativa en nuestro país, la formación docente articulada con el uso de las TIC se encuentra aún en una etapa incipiente en la que ha prevalecido la habilitación metodológica e instrumental sobre la base de un dominio de contenidos disciplinarios específicos.

En el caso de la UNAM, a lo largo de poco más de cuatro décadas, diversas entidades han representado un soporte importante en este rubro, mediante la oferta diversificada de especializaciones, diplomados, cursos, talleres y laboratorios para la formación y actualización de docentes, tanto de la propia institución como de otras instituciones nacionales y extranjeras. Concretamente, desde la década pasada la Coordinación de Universidad Abierta y Educación a Distancia (CUAED) ha venido ofreciendo este tipo de soporte con especial énfasis en el uso de las TIC.

En relación con lo anterior, el proyecto que aquí se presenta tiene como propósito central la preparación de un estado del conocimiento que caracterice un panorama de corte comparativo sobre la práctica docente de los profesores del SUAyED/FFyL, destacando en particular los procesos de incorporación y manejo de las TIC en el trabajo docente cotidiano 


\section{INVESTIGACIÓN COMPARADA EN EDUCACIÓN: PROCESOS DE INCORPORACIÓN DE LAS \\ TIC EN LA PRÁCTICA DOCENTE DEL SUAYED-FFYL UNAM \\ DOI: http://dx.doi.org/10.5007/1983-4535.2012v5n4p188}

en condiciones de no presencialidad (enseñanza abierta, enseñanza virtual), a fin de analizar aportes, ventajas y limitaciones de esta tendencia actual.

Un aspecto nodal de esta investigación consiste en ubicar, comparar y analizar las especificidades en cuanto a la incorporación y el manejo de las TIC por parte de los profesores de las diversas Licenciaturas que conforman la planta académica de la División SUAyED/FFyL. Estimamos que los resultados de este trabajo representarán un aporte de base para la toma de decisiones posteriores relacionadas con aspectos como contratación de personal, procesos de actualización, productividad y evaluación académicas, proyectos de trabajo colegiado, entre otros. Asimismo, consideramos que todo lo anterior redundará de manera definitiva en favor del rendimiento académico de los estudiantes universitarios de esta modalidad educativa.

\section{PREGUNTA PRINCIPAL}

¿Qué semejanzas y diferencias caracterizan la práctica docente cotidiana de los profesores de las Licenciaturas de la División SUAyED/FFyL, a partir de los procesos de incorporación y utilización las TIC?

De esta pregunta se desprenden las siguientes cuestiones:

¿Cuál es el perfil académico-pedagógico de la planta docente de la citada División?

¿Cuáles son las semejanzas y diferencias entre cada una de las Licenciaturas?

¿Cómo se caracteriza la práctica docente de los profesores de esta entidad institucional?

¿Cuáles son las herramientas tecnológicas más utilizadas en el trabajo cotidiano con los alumnos, en las diversas Licenciaturas del SUAyED/FFyL?

¿A partir de cuáles referentes teórico-metodológicos incorporan los docentes el uso de las TIC en esta modalidad?

¿De qué manera ha influido la incorporación y manejo de las TIC en el despliegue de estrategias didácticas por parte de los docentes?

¿Cuál es la perspectiva de los profesores respecto de los aportes, las ventajas y limitaciones de la presencia de las TIC en el desarrollo de la práctica docente en las diversas Licenciaturas de esta modalidad educativa?

\section{PLANTEAMIENTO Y JUSTIFICACIÓN DEL PROBLEMA}

Desde su surgimiento a la fecha, la definición del perfil del profesor en la entidad de referencia ha sido el resultado de una construcción basada en elementos conceptuales y 


\section{INVESTIGACIÓN COMPARADA EN EDUCACIÓN: PROCESOS DE INCORPORACIÓN DE LAS \\ TIC EN LA PRÁCTICA DOCENTE DEL SUAYED-FFYL UNAM \\ DOI: http://dx.doi.org/10.5007/1983-4535.2012v5n4p188}

operativos sobre la docencia. A partir de definiciones básicas sobre el aprendizaje y el estudio independientes, así como la relación pedagógica alternativa para un contexto diferente al de la clase presencial, podemos rastrear algunos de los rasgos bajo las siguientes caracterizaciones oficializadas en momentos específicos (Rojas, 2010):

a) Perfil docente de 1977: El tutor proporciona asesoría personal a los alumnos que enfrentan dificultades en el manejo de los materiales.

b) Perfil docente de 1982: El tutor cumple funciones de coordinador de grupos de aprendizaje, pues además de atender la transmisión de la información cuida que todos los alumnos participen y manejen los instrumentos de indagación y de acción sobre objetos de conocimiento.

c) Perfil docente de 1993: El tutor tiene tres funciones básicas: facilitar la adaptación del alumno a la modalidad, orientarlo para aprovechar los recursos disponibles y apoyar el estudio independiente.

d) Perfil docente de 2008: El tutor es un experto quien, además del manejo de las estrategias para el trabajo en la modalidad abierta y a distancia, posee habilidades para el uso educativo de las tecnologías de información y comunicación.

Observamos aquí una serie de cambios hasta llegar a un perfil que se ha ampliado gradualmente con el añadido de convertir al profesor en el asesor responsable de la utilización de recursos tecnológicos avanzados para una mayor cobertura de servicios educativos. Se trata de una sumatoria de nuevas habilidades tecnológicas que habrán de incorporarse al bagaje disciplinario y didáctico requerido, de nuevas "competencias" docentes, aun cuando institucionalmente no se cuente todavía con una delimitación por competencias ex profeso, en este caso, ni de la formación docente ni del perfil profesional del docente para esta modalidad.

Ahora bien, a modo de destacar los aportes del proyecto propuesto hemos definido tres cuestionamientos a partir de los cuales ubicamos los rasgos que caracterizan tanto el trabajo a desarrollar como los resultados por obtener.

1. ¿Qué queremos investigar?

Abordaremos el desarrollo de la práctica docente de los profesores de las diversas Licenciaturas que componen la citada entidad (Filosofía, Historia, Letras Hispánicas, Letras Inglesas, Geografía, Pedagogía y Bibliotecología), mediante la construcción de un encuadre multinivel que comprenda, por una parte, el contexto sociohistórico del espacio institucional y, por otra parte, las especificidades de la práctica docente en la División SUAyED/FFyL. 


\section{INVESTIGACIÓN COMPARADA EN EDUCACIÓN: PROCESOS DE INCORPORACIÓN DE LAS \\ TIC EN LA PRÁCTICA DOCENTE DEL SUAYED-FFYL UNAM \\ DOI: http://dx.doi.org/10.5007/1983-4535.2012v5n4p188}

Para cumplir este propósito habremos de caracterizar la práctica docente como primera fuente de análisis, ubicando el perfil académico, pedagógico y de manejo de las TIC.

2. ¿Por qué queremos investigar este tema?

Actualmente, el uso de las TIC en el desarrollo de la práctica docente es una constante que ha transformado de manera definitiva los estilos de docencia, el despliegue de estrategias didácticas, la articulación de otros recursos e insumos, y lo más importante, las formas de comunicación e interacción entre docentes y alumnos en el contexto de la vida académica e institucional. Durante las dos últimas décadas, organismos nacionales e internacionales lo mismo que comunidades de investigadores de diferentes países sensibles a esta problemática, han revelado el potencial, así como los alcances, las ventajas y limitaciones de esta nueva tendencia en la práctica docente a través de publicaciones diversas (Rojas, 2011).

Sin embargo aún son escasos los estudios sobre el tema que nos ocupa, toda vez que las búsquedas efectuadas reflejan como objeto de investigación recurrente el de la formación inicial y la actualización docente en el nivel superior en vinculación con el manejo de las TIC y la formación de asesores. Un esbozo panorámico de los temas abordados en los diferentes documentos encontrados, algunos de ellos con un tratamiento de corte prescriptivo, permite una primera indicación que sitúa la formación como el eje de la investigación distinguiendo al alumno y al docente en tanto sujetos a formar para el manejo de la TIC. Por otra parte, también salta a la vista como un eje articulante el tema de la ampliación de servicios educativos en las modalidades no presenciales, siendo éste un factor que obliga a replantear el uso de las TIC en el ámbito educativo.

Con base en lo anterior y ante la carencia de un estudio de la índole propuesta, estimamos como tarea de importancia la elaboración de un estado de conocimiento de corte comparativo sobre la práctica docente de los profesores de la División SUAyED/FFyL. Su relevancia radica en que el documento a realizar contará con un análisis detallado de los procesos de incorporación y manejo de las TIC, mostrando en contraste las características, semejanzas y diferencias en este rubro específico, en los perfiles docentes de las plantas académicas de cada una de las Licenciaturas de la entidad en cuestión. 


\section{INVESTIGACIÓN COMPARADA EN EDUCACIÓN: PROCESOS DE INCORPORACIÓN DE LAS \\ TIC EN LA PRÁCTICA DOCENTE DEL SUAYED-FFYL UNAM \\ DOI: http://dx.doi.org/10.5007/1983-4535.2012v5n4p188}

3. ¿Qué podemos aportar con la investigación?

Además de los rasgos ya indicados, el hecho de disponer de un estado de conocimiento con una base de datos ampliada sobre el perfil de los docentes, enmarcado en una perspectiva comparativa sobre el manejo de las TIC en la práctica docente, representará un avance sustantivo en el conocimiento de la vida académica en nuestra entidad. El documento en cuestión contendrá información detallada a modo de un catálogo con referentes básicos para apoyar la toma de decisiones en rubros tales como: contratación de personal, programas de formación y actualización de docentes, proyectos académicos e investigativos de trabajo colegiado, índices productividad, lineamientos para la evaluación académica, entre otros.

En una visión conjunta, partimos de la premisa de caracterizar la práctica docente en vinculación con las condiciones de formación y profesionalización de la planta académica de nuestra entidad. El abanico de aspectos a abordar sería el siguiente: contexto de la formación, manejo de contenidos académicos específicos, bases psicopedagógicas de la docencia, marco curricular de la formación académica, utilización de las TIC en la práctica educativa, estrategias de aprendizaje y evaluación.

Otra condición vinculada con la caracterización del perfil de los docentes de la entidad, consistirá en partir de la base de la aproximación comparativa como un ejercicio de investigación complejo para abordar de manera articulada las tareas de formación y profesionalización de los docentes. Por ello insistiremos en presentar nuestra investigación en términos de un proceso de valoración que, sin prescindir del manejo de instrumentos metodológicos y técnicos, recupere el encuadre de una contextualización sociohistórica en un marco institucional, a la vez que influya para la impulsar la participación de los docentes en los procesos de incorporación y manejo de las TIC en el trabajo cotidiano.

\section{OBJETIVOS}

El presente proyecto tiene como objetivo principal el siguiente:

Elaborar un estado de conocimiento de corte comparativo sobre la práctica docente de los profesores de la División SUAyED/FFyL, tomando en cuenta la incorporación y el manejo de las nuevas tecnologías de la información y la comunicación en modalidades de enseñanza no presenciales (enseñanza abierta, enseñanza virtual). 
Para su cumplimiento proponemos los objetivos específicos enunciados a continuación:

a) Estructurar el encuadre teórico-metodológico ad hoc derivado de la realización de y/o participación en actividades académicas específicas (investigación documental, seminarios de estudio, eventos especializados, elaboración de textos).

Elaborar un diagnóstico nacional sobre los sistemas de universidad abierta y a distancia en México, a modo de contextualización de la oferta educativa en esta modalidad en la UNAM.

b) Caracterizar de manera comparativa la práctica docente de los profesores de las Licenciaturas de la División SUAyED/FFyL a partir de un estudio diagnóstico, destacando lo referente a la incorporación y el manejo de las TIC.

c) Analizar el impacto de las TIC en el despliegue de estrategias didácticas de los profesores para la construcción de la práctica docente cotidiana.

d) Valorar los aportes, ventajas y limitaciones de la presencia de las TIC en el desarrollo de la docencia en esta modalidad educativa.

e) Colaborar, mediante la organización y/o participación, en eventos institucionales, nacionales e internacionales con la presentación de avances y resultados de investigación.

f) Contribuir en la formación de recursos humanos de egresados de licenciatura, con apoyos para elaboración de tesis y realización de estudios de posgrado.

g) Preparar el documento final mediante la integración de los documentos previos de discusión teórico-analítica y los reportes parciales de investigación.

\section{HIPÓTESIS}

El proyecto propuesto parte del siguiente cuestionamiento:

Respecto del manejo de las TIC, ¿hay diferencias sustantivas en la práctica docente entre los profesores de las Licenciaturas del SUAyED/FFyL?

A partir de esta pregunta y con base en investigaciones precedentes (Rojas, 2010), formulamos las argumentaciones siguientes:

a) Si bien las propuestas de formación y actualización docentes de los profesores del SUAyED/FFyL han correspondido con los requerimientos institucionales en diferentes momentos del desarrollo de la entidad, observamos que aún prevalece una preparación insuficiente en cuanto al manejo de las TIC.

b) Por otra parte, la construcción de la práctica docente no puede circunscribirse a aspectos técnicos pues reduciría la docencia a una actividad mecánica. Aun así, es 
ineludible la necesidad de preparar a los profesores para el uso didáctico de las TIC en la práctica educativa.

c) En este sentido reiteramos la pertinencia de realizar esta investigación a fin de comparar las especificidades sobre el manejo de las TIC por parte de los profesores de las diversas Licenciaturas del SUAyED/FFyL.

d) Con base en lo anterior, consideramos que la elaboración de un estado del conocimiento de corte comparativo que caracterice la práctica docente en esta entidad, destacando los procesos de incorporación y manejo de las TIC en la práctica docente, aportará información clave para la toma de decisiones relacionada con aspectos como contratación de personal, procesos de actualización, proyectos de trabajo colegiado, productividad y evaluación académica, entre otros.

e) Finalmente, la consulta de este documento redundará en la implantación de estrategias institucionales favorables para un mejor rendimiento académico de los estudiantes en esta modalidad educativa.

\section{ESTADO DEL ARTE}

El surgimiento del Sistema Universidad Abierta (SUA) en nuestro país data de principios de los años setenta. Concretamente, en el año de 1972 se instauró en la UNAM en una coyuntura histórica donde convergieron por una parte, las estrategias gubernamentales de modernización político-económica, y por otra, los proyectos de corte reformista para la modernización académica de las instituciones universitarias. De hecho, su creación representó una propuesta formativa sin precedente en la educación superior en México, cuyos objetivos comunes fueron: a) reducir la necesidad de la escolarización para favorecer el acceso a la educación de estudiantes en circunstancias diferentes a las usuales, en ámbitos extraescolares o en combinación con las instituciones; $\mathrm{y}, \mathrm{b}$ ) basarse en la potencialidad del alumno para estudiar y aprender por cuenta propia. (Rojas, 1998). La importancia y expansión de dicho sistema se debe, sin duda, a la invaluable alternativa que ha representado el hecho de satisfacer las necesidades y demandas formativas a través de modalidades no presenciales y de la utilización de innovaciones tecnológicas y comunicacionales.

A más de cuatro décadas de su implantación, se cuenta con una amplia experiencia respecto de la organización y operación de este tipo de modalidad, situación que contrasta con el desarrollo poco consistente de programas para la formación de especialistas y, en particular, de docentes en este campo. De hecho, el personal docente incorporado a los sistemas abiertos y a distancia proviene, en su mayoría, de los sistemas escolarizados. Esto hace evidente cómo mediante la práctica educativa se posibilita el desarrollo de habilidades que permiten a este 


\section{INVESTIGACIÓN COMPARADA EN EDUCACIÓN: PROCESOS DE INCORPORACIÓN DE LAS \\ TIC EN LA PRÁCTICA DOCENTE DEL SUAYED-FFYL UNAM \\ DOI: http://dx.doi.org/10.5007/1983-4535.2012v5n4p188}

tipo de profesores el cumplimiento de las funciones docentes propias de la citada modalidad educativa.

En cuanto a la docencia, ésta constituye una actividad compleja vinculada con diversos ámbitos (social, institucional, grupal e individual). Los problemas a los que se enfrenta todo docente en el aula son un reflejo de aquéllos que afectan a la sociedad en general y a las instituciones en particular.

Ahora bien, en relación con la categorización de la práctica docente se observan dos tendencias. En la primera, esta actividad está considerada como un acto técnico de aplicación de procedimientos y recursos para la enseñanza, es decir, con una orientación instrumentalista. En la segunda tendencia, la docencia es vista como una profesión dado el reconocimiento de sus dimensiones de trabajo que van más allá de preparar e impartir clases, calificar tareas y exámenes, situando al profesor como un intelectual que dialoga e interactúa con objetos de conocimiento (Carr, 1998).

Por otra parte, desde principios de la década de los setenta y hasta la fecha, las necesidades y demandas planteadas por las instituciones de educación superior de incorporación y preparación de personal para el desempeño de la docencia, han permitido consolidar programas de formación docente orientados a la profesionalización. Desde esta perspectiva, la institucionalización de la formación docente en el nivel universitario ha derivado en una formación doble (Ferry, 1990): a) el oficio de profesor que exige una formación académico-disciplinaria, $\mathrm{y}, \mathrm{b}$ ) una formación profesional acotada en el mejor de los casos a una habilitación pedagógica, entreverada de aspectos vinculados con tareas de concertación, gestión y orientación, entre otras.

En este sentido se enfatiza que la formación pedagógica puede plantearse con diferentes enfoques, que van desde un reduccionismo meramente técnico hasta la investigación sobre la práctica docente. Así, es posible argumentar que en este proceso las renovaciones técnicas son efímeras si no van acompañadas de la reflexión acerca de la relación interpersonal y las actitudes necesarias para favorecer el crecimiento personal del docente y de sus alumnos. Más aún, este panorama caracterizado tan a grandes rasgos se entreteje en una perspectiva más amplia y compleja a la cual algunos teóricos han denominado Aldea global (McLuhan, 1986), Era Tecnotrónica (Zbigniew, 1973), Sociedad Postindustrial (Bell, 2006), Sociedad de la Información (Castell, 2002), Sociedad del Conocimiento (Drucker, 1994), inter alia. Todas estas denominaciones epocales tienen en 


\section{INVESTIGACIÓN COMPARADA EN EDUCACIÓN: PROCESOS DE INCORPORACIÓN DE LAS \\ TIC EN LA PRÁCTICA DOCENTE DEL SUAYED-FFYL UNAM \\ DOI: http://dx.doi.org/10.5007/1983-4535.2012v5n4p188}

común la idea de que las TIC pueden mejorar la vida de todos los habitantes del planeta. Sin duda, en el contexto educativo las TIC brindan numerosos apoyos para hacer más dinámico y flexible el proceso formativo, considerando que se enfrentan nuevas formas de interacción en ambientes de aprendizaje no presenciales, donde prevalece una comunicación mediada por los recursos tecnológicos, lo que ha generado múltiples retos para la formación de los seres humanos en general.

En este escenario, al considerar la vertiginosidad de los avances tecnológicos y su consecuente impacto en el ámbito educativo, se vuelve obligado el tema de la formación y el desempeño docentes de todos los niveles y modalidades educativos a la luz de nuevos parámetros. Destaca también el tema de los estándares de competencias en TIC para docentes, y a este respecto la UNESCO (2008) señala que en un contexto educativo sólido las TIC posibilitan la adquisición y el desarrollo de competencias informacionales, sustentables sobre la base de una formación docente no sólo disciplinaria y pedagógica, sino además de una actualización tecnológica o de alfabetización digital básica.

En este sentido, resulta indispensable que el profesor maneje diversas herramientas tecnológicas para apoyar los procesos de enseñanza y aprendizaje; esto último en el supuesto de que la incorporación de estos medios y su adecuada utilización didáctica deriven en una formación académica más consolidada, pensando -por ejemplo- en el uso de sitios web, redes sociales, blogs, plataformas educativas, webquest y portafolio electrónico, por mencionar algunas de estas herramientas.

En resumen, el uso didáctico de las TIC coloca un fuerte debate sobre el papel de los docentes en la escuela, al discutir el pasaje entre el modelo de aula tradicional, centrado en la enseñanza y en el profesor con recursos elementales, al de un modelo centrado en el estudiante y en el aprendizaje, perfilando con ello el papel del profesor visto como un mediador. En el caso específico del SUAyED/FFyL (Vera, 2005; Rojas, 2010), en la construcción de la práctica docente cotidiana, el profesor ha tenido que adaptarse a una situación educativa diferente en la cual su tarea principal no es la de "impartir clases", y en la que tampoco los alumnos están obligados a asistir regularmente dado que cuentan con materiales para el estudio independiente. Las actividades del docente en esta modalidad se han centrado en brindar apoyo académico a los alumnos acerca de aspectos diversos: uso de guías y textos, desarrollo de estrategias de estudio, aclaración de dudas, así como propuestas 


\section{INVESTIGACIÓN COMPARADA EN EDUCACIÓN: PROCESOS DE INCORPORACIÓN DE LAS \\ TIC EN LA PRÁCTICA DOCENTE DEL SUAYED-FFYL UNAM \\ DOI: http://dx.doi.org/10.5007/1983-4535.2012v5n4p188}

para la organización de grupos de trabajo, intercambio de ideas y procedimientos de evaluación, entre otras.

Respecto de la estrategia institucional para la formación docente en esta entidad (Rojas, 2010), desde 1977 y hasta 1990 se contó con la Unidad de Asesoría Pedagógica para la impartición de cursos anuales a los profesores adscritos al sistema. De 1992 a 2000 los apoyos para la formación y actualización docentes se centraron en una oferta de cursos, seminarios, talleres y diplomados. A partir 2005, la Coordinación de Universidad Abierta y Educación a Distancia (CUAED) de la UNAM quedó a cargo de la actualización docente mediante una cartera temática de cursos orientados básicamente al manejo de plataformas electrónicas como espacios virtuales de enseñanza y aprendizaje, la asesoría en línea y la utilización de las TIC en el estudio a distancia. Sin embargo, se estima que a pesar de la oferta descrita, la actualización de corte tecnológico es diferente en cada una de las Licenciaturas del SUAyED, y por consiguiente la práctica docente observa rasgos específicos.

\section{METODOLOGÍA}

El presente proyecto tiene como encuadre teórico-metodológico el de la investigación educativa comparada y estará vinculado con los trabajos del Seminario Interinstitucional Permanente de Educación Comparada (SIPEC). ${ }^{1}$ Cabe señalar que en las últimas décadas la investigación comparada se ha proyectado como un ámbito teórico y metodológico cada vez más importante, sobre todo a partir de los vertiginosos procesos de internacionalización y globalización. Entendida como perspectiva de estudio, la investigación comparada ofrece abordajes analíticos de sistemas diversos (p. ej., económicos, políticos, jurídicos, educativos, tecnológicos, etcétera), los cuales han posibilitado ubicar semejanzas y diferencias entre entidades, regiones, o bien, países, para así detectar problemas comunes y soluciones probadas.

Coincidimos con los expertos en el tema (Cf. Altbach, Schriewer, inter alia) en que, mediante los abordajes comparativos en un campo de estudio específico, como lo es el educativo, es posible identificar tendencias y procesos de convergencia y divergencia. Finalmente, a través de la investigación comparada se han difundido y retroalimentado los

\footnotetext{
${ }^{1}$ Seminario a cargo de la Dra. en C. Ileana Rojas Moreno y la Mtra. en C. Zaira Navarrete Cazales, registrado en el Centro de Apoyo a la Investigación de la Facultad de Filosofía y Letras de la UNAM (N PIFFyL 2006005 ). La información detallada se localiza en la siguiente liga: http://www.filos.unam.mx/educomparada/
} 


\section{INVESTIGACIÓN COMPARADA EN EDUCACIÓN: PROCESOS DE INCORPORACIÓN DE LAS \\ TIC EN LA PRÁCTICA DOCENTE DEL SUAYED-FFYL UNAM \\ DOI: http://dx.doi.org/10.5007/1983-4535.2012v5n4p188}

acuerdos derivados de reuniones y conferencias internacionales sobre distintos aspectos de los procesos sociales.

Con base en este referente y para efectos de la investigación propuesta, la metodología de aproximación comparada se utilizará como la perspectiva de abordaje del objeto que nos ocupa. En este sentido retomamos uno de los argumentos de Schriewer (2002), al enfatizar que la investigación comparada comprende tanto un marco conceptual y metodológico como un trabajo de análisis sociohistórico. ${ }^{2}$ Por tanto, siguiendo este planteamiento encontramos que todos los ámbitos de estudio en el marco de una designación común (en este caso el campo educativo), abarcan tipos divergentes de teorías (científicas, de reflexión) relacionadas con orientaciones funcionales y la definición concomitante de problemas de diferentes subsistemas de la sociedad.

Por cuanto a los enfoques de las estrategias teórico-metodológicas a utilizar, combinaremos de manera justificada tratamientos y procedimientos tanto de corte cuantitativo como cualitativo, atendiendo al ordenamiento propuesto en la obtención de productos indicados en los objetivos específicos del proyecto. Así, cubriremos siete líneas de acción tipificadas de la siguiente manera:

1) Línea de investigación documental.- Esta línea acompañará todo el proceso de la investigación y comprenderá la integración sistemática (descriptiva y analítica) de un acervo informativo, a través de la recopilación de materiales vinculados con el tema, obtenidos de fuentes bibliográficas, hemerográficas y documentales, y localizados en bibliotecas especializadas, o bien, mediante catálogos electrónicos.

2) Línea de discusión teórico-metodológica.- En esta línea se realizarán tres seminarios de estudio con una duración anual, distribuidos en temáticas específicas.

\footnotetext{
${ }^{2}$ De acuerdo con Schriewer (2002), los contextos sociales (locales, nacionales, internacionales, globales) ejercen una influencia decisiva sobre fenómenos internos en ámbitos más acotados. Tal es el caso del campo educativo al considerar los procesos de la educación, las organizaciones de los sistemas educativos, los modelos de interacción pedagógica o las tradiciones del pensamiento educativo, así como sobre sus efectos sociales y problemas resultantes. Bajo esta lógica, dichos contextos sociales, que se presuponen eficaces, se pueden descomponer en variables contextuales particulares (variables explicativas), según intereses teóricos determinados.

En este sentido encontramos que la ampliación de la base experiencial (inter-nacional, etc.) despliega, a través de la comparación, un campo de observación, desarrollado histórica y socialmente, que contiene en sí mismo estas variables condicionantes en diversas intensidades, y que, en ese sentido, hace analizable de forma ordenada el poder efectivo de esas variables con miras a identificar la relación de explicación existente entre las condiciones contextuales (societales, nacionales, etc.) y los fenómenos educativos intra-societales. Lo anterior como parte del despliegue y aplicación de diversos procedimientos metodológicos para la construcción de categorías de análisis y el desarrollo de la contextualización de los casos a comparar.
} 


\section{INVESTIGACIÓN COMPARADA EN EDUCACIÓN: PROCESOS DE INCORPORACIÓN DE LAS \\ TIC EN LA PRÁCTICA DOCENTE DEL SUAYED-FFYL UNAM \\ DOI: http://dx.doi.org/10.5007/1983-4535.2012v5n4p188}

En el primer seminario (2013) se analizarán cuestiones teóricas y metodológicas de la investigación comparada a partir de la discusión de textos de autores reconocidos (Schriewer, Altbach, entre otros), a fin de estructurar el encuadre sociohistórico, definir los niveles de comparatividad de la investigación, ${ }^{3}$ las categorías de análisis, los cuadros de tipologías, el universo de trabajo, el diseño de instrumentos de encuesta y entrevista y el piloteo respectivo. También se abordarán aspectos metodológicos para el arranque del diagnóstico nacional sobre oferta de estudios de licenciatura en modalidad no presencial.

En el segundo seminario (2014) se continuarán abordando cuestiones a propósito de estrategias metodológicas y técnicas para la recolección de información mediante la aplicación de cuestionarios, la realización de entrevistas y lineamientos generales para la sistematización de volúmenes de datos. Asimismo, se considerarán los lineamientos para la estructura del primer libro a publicar.

En el tercer seminario (2015) se desarrollarán aspectos de procesamiento de información, elaboración de reportes y documentos parciales, estructura de textos a publicar (artículo de revista y libro con resultados finales). Este espacio permitirá abordar nuevamente consideraciones teórico-metodológicas, a modo de afinar la presentación final de los documentos que se enviarán a dictamen para su publicación.

3) Línea de trabajo de campo.- En esta línea se incluye lo concerniente a la preparación de los instrumentos a utilizar (encuesta, entrevista) en cuanto a su diseño, piloteo previo, ajustes y aplicación a la población-universo. Se consideran también tareas como trascripción de entrevistas y preparación de archivos documentales para resguardar la información recopilada.

4) Línea de sistematización de la información.- En esta línea están consideradas todas aquellas actividades de trabajo con la información recopilada mediante la elaboración cuadros comparativos, esquemas, marcos conceptuales y cuadros de vaciado que nos permitirán

\footnotetext{
${ }^{3}$ De acuerdo con la diferenciación propuesta por Schriewer (1993) ubicamos dos formas de comparatividad aplicables en la investigación. La primera es la comparación simple o de nivel único, procedimiento consistente en relacionar objetos a comparar, considerando solamente sus aspectos observables; en este primer nivel sólo se realiza un análisis por campos de características homólogas, y alcanza un avance de descripción básico de la información. La segunda consiste en la comparación compleja o de niveles múltiples, procedimiento mediante el cual se abordan las relaciones que pueden existir entre los distintos fenómenos, variables o niveles de sistemas. Para ello se inicia con articulaciones hipotéticas entre variables identificadas a diferentes niveles de análisis. Enseguida se procede a identificar sus manifestaciones empíricas observables como grupos de fenómenos variables, incorporados en diversos contextos socioculturales. Posteriormente, se trata luego de vincular estas relaciones empíricas una con otras, a la par que con las hipótesis de investigación formuladas previamente, para examinar su capacidad de generalización teórica y de explicación. De hecho, es esta vertiente la que nos interesa destacar, insistiendo en la importancia de la comparatividad en las ciencias sociales.
} 


\section{INVESTIGACIÓN COMPARADA EN EDUCACIÓN: PROCESOS DE INCORPORACIÓN DE LAS \\ TIC EN LA PRÁCTICA DOCENTE DEL SUAYED-FFYL UNAM \\ DOI: http://dx.doi.org/10.5007/1983-4535.2012v5n4p188}

organizar, analizar y sistematizar los paquetes de datos obtenidos, integrándolos en bases de datos específicas.

5) Línea de difusión académica.- Para esta línea del proyecto estamos programando actividades tanto de participación en eventos nacionales e internacionales, como de organización de eventos interinstitucionales. Consideramos de vital importancia atender la tarea de difusión académica a lo largo del proceso y con los avances reportados, hasta llegar a la integración de los productos totales (dos libros y un artículo especializado). Los espacios de difusión de resultados son los siguientes:

Primer año (2013): a) Evento internacional "XV Comparative Education World Congress", a realizarse en la Ciudad de Buenos Aires, Argentina del 24 al 28 de junio. Se participará con ponencia. b) Organización de evento institucional en la modalidad de conferencia para presentar el proyecto y los avances de investigación con título por definir; en este caso la sede será la propia Facultad de Filosofía y Letras en la primera quincena del mes de octubre. c) Evento nacional "XII Congreso Nacional de Investigación Educativa", organizado por el Consejo Mexicano de Investigación Educativa en la primer semana del mes de noviembre, y con sede por definir. Se participará con ponencia.

Segundo año (2014): a) Evento internacional "XIV Congreso Nacional de Educación Comparada", a realizarse en España en el mes de octubre, con sede y fecha específica por definir. Se participará con ponencia. b) Organización del evento institucional "Coloquio: Uso de TIC en la enseñanza universitaria" para presentar los avances de investigación del período; en este caso la sede será por segunda ocasión la Facultad de Filosofía y Letras, y la fecha estimada se propondrá para la primera quincena del mes de noviembre.

Tercer año (2015): a) Evento internacional "2015 Annual Meeting” de la American Educational Research Association, en los USA (abril, a realizarse en los Estados Unidos de Norteamérica en el mes de abril, con sede y fecha específica por definir. Se participará con ponencia. b) Organización de evento interinstitucional en la modalidad de Seminario Especializado para presentar los productos de investigación y se contará con la invitación de un expositor experto en TIC aunque el título quedará por definirse; en este caso la sede será la propia Facultad de Filosofía y Letras en la primera quincena del mes de octubre. c) Evento nacional "XIII Congreso Nacional de Investigación Educativa", organizado por el Consejo Mexicano de Investigación Educativa en la primer semana del mes de noviembre, y con sede por definir. Se participará con ponencia. 


\section{INVESTIGACIÓN COMPARADA EN EDUCACIÓN: PROCESOS DE INCORPORACIÓN DE LAS \\ TIC EN LA PRÁCTICA DOCENTE DEL SUAYED-FFYL UNAM \\ DOI: http://dx.doi.org/10.5007/1983-4535.2012v5n4p188}

6) Línea de formación de recursos humanos.- Hemos cuidado que para la puesta en marcha del proyecto, durante el primer año se cuente con la participación de una estudiante egresada de la Licenciatura en Pedagogía de la División SUAyED, contando con este espacio para la elaboración de tesis de licenciatura. Asimismo, estimamos que para los dos años restantes podrán incorporarse uno o dos egresados más a fin de colaborar en los trabajos de campo, organización de actividades de difusión y apoyo en las tareas de edición de documentos. Muy probablemente se solicite incluso el soporte para estudios de posgrado de alguno de los participantes Consideramos que la posibilidad de ofrecer un espacio formativo en el quehacer investigativo y, si es el caso, de una compensación económica, será uno de los resultados de mayor impacto.

7) Línea de producción de obra.- Además de los documentos enunciados en las líneas anteriores tales como reportes parciales, avances de resultados y ponencias para presentación en eventos especializados, en esta última línea estamos considerando la presentación de tres productos para publicación, previa dictaminación rigurosa de comités editoriales.

El primer documento a presentar en octubre de 2014 será el libro "Diagnóstico nacional sobre los sistemas de universidad abierta y a distancia en México", material que reunirá un panorama analítico sobre las diferentes instituciones de educación superior (IES), trátese de universidades, centros y/o institutos, con una caracterización detallada sobre la oferta educativa en esta modalidad. La publicación quedará a cargo de la Facultad de Filosofía y Letras.

El segundo documento será un artículo sobre avances y resultados preliminares de la investigación propuesta, mismo que se enviará en junio de 2015 a la Revista Mexicana de Investigación Educativa del COMIE.

El tercer documento será el estado de conocimiento en el formato de libro tentativamente denominado "Presencia de las TIC en la práctica docente en el SUAyED/FFyL de la UNAM. Un estudio comparativo". La entrega de este texto marcará el cierre del proyecto y su presentación está programada para octubre de 2015, considerando la propuesta de una coedición entre la Facultad de Filosofía y Letras y la Editorial Plaza y Valdés.

Por último, y en relación con las argumentaciones puntualizadas en el rubro de "Hipótesis" del presente documento, enfatizamos el hecho de contar con trabajos precedentes que respaldan tanto el conocimiento del objeto de investigación (Rojas, 2010), como de la 
estrategia metodológica propuesta (Rojas y Navarrete, 2010), principalmente. ${ }^{4}$ Por ello consideramos que el manejo de estos referentes, entre otros de los indicados en los diversos productos que dan cuenta de la trayectoria académica y de investigación del grupo de trabajo, representan los elementos de base que garantizarán el logro de objetivos y metas propuestos.

\section{DISEÑO DE INVESTIGACIÓN}

Las metas que, en términos de productos, se lograrán con el desarrollo del presente proyecto se distribuirán de la siguiente manera:

Primer año (2013). Meta: Elaboración de marco teórico-metodológico e instrumentos de recolección de información. Actividades:

1) Seminario de discusión teórico-metodológica (feb-mayo).

2) Diseño de instrumentos de encuesta y entrevista para trabajo de campo (agostonov).

3) Elaboración de diagnóstico nacional sobre oferta de estudios de licenciatura en modalidad no presencial (feb-nov).

4) Ponencia para "XV Comparative Education World Congress", en Argentina (junio).

5) Organización de conferencia para presentación de proyecto en la FFyL-UNAM (oct).

6) Ponencia para "XII Congreso Nacional de Investigación Educativa", en México, (noviembre).

Segundo año (2014). Meta: Realización de trabajo de campo. Actividades:

1) Seminario de apoyo al trabajo de campo (feb-nov).

2) Aplicación de cuestionarios, realización de entrevistas, avances de sistematización de la información recabada (feb-nov).

3) Entrega del "Diagnóstico nacional sobre los sistemas de universidad abierta y a distancia en México" para su publicación como libro (oct).

4) Ponencia para XIV Congreso Nacional de Educación Comparada, en España (oct).

5) Organización del Coloquio "Uso de TIC en la enseñanza universitaria” en la FFyLUNAM (nov).

\footnotetext{
${ }^{4}$ Los documentos de referencia están citados en la bibliografía de este proyecto de investigación. 


\section{INVESTIGACIÓN COMPARADA EN EDUCACIÓN: PROCESOS DE INCORPORACIÓN DE LAS \\ TIC EN LA PRÁCTICA DOCENTE DEL SUAYED-FFYL UNAM \\ DOI: http://dx.doi.org/10.5007/1983-4535.2012v5n4p188}

Tercer año (2015). Meta: Conclusión y divulgación de la investigación. Actividades:

1) Seminario para procesamiento de información (feb-nov).

2) Ponencia para "2015 Annual Meeting" de la American Educational Research Association, en los USA (abril).

3) Envío de artículo para publicación en Revista del COMIE (junio).

4) Entrega de libro como producto de investigación (oct).

5) Organización de evento interinstitucional en la FFyL-UNAM para difusión de resultados con invitación de expositor experto en TIC (oct).

6) Ponencia en "XIII Congreso Nacional de Investigación Educativa", en México (nov).

\section{REFERENCIAS CONSULTADAS}

Adell, J. (1997). Tendencias en la sociedad de las tecnologías de la información, EDUTEC, Revista Electrónica de Tecnología Educativa, No. 7. Fecha de consulta: 12/04/2004, en http://edutec.rediris.es/documentos/1997/tndencias.html

Aguaded, J. y J. Cabero, (2002), Educar en red. Internet como recurso para la educación, España, Ediciones ALJIBE

Altbach, P. y G. Kelly (1990), Nuevos enfoques en educación comparada, Madrid, Mondadori.

Amador B., R. (2006), La universidad en Red. Un nuevo paradigma de la educación superior, en Revista Mexicana de Investigación Educativa, México: COMIE/UAM, 2004.1. Vol. XI, N² 28 pp.155-177.

Área, M. (2008). ¿Las TIC están generando innovación pedagógica en las aulas? Lo que nos dice la investigación. Ordenadores en el aula. Blog sobre los retos de la educación ante la tecnología y la cultura digital. Fecha de consulta 20/01/2011 en:

http://ordenadoresenelaula.blogspot.com/2 08/01/las-tic-estn-generandoinnovacin.html

Bell, Daniel (2006), El advenimiento de la sociedad post-industrial, Buenos Aires, Alianza Editorial.

Benítez G., R. (2007). "Formación docente para educación a distancia", tesis de maestría, México, UNAM-FFyL.

Cabero, J. (2006). Nuevas tecnologías aplicadas a la educación. España, McGraw-

Hill/Interamericana. 


\section{INVESTIGACIÓN COMPARADA EN EDUCACIÓN: PROCESOS DE INCORPORACIÓN DE LAS \\ TIC EN LA PRÁCTICA DOCENTE DEL SUAYED-FFYL UNAM \\ DOI: http://dx.doi.org/10.5007/1983-4535.2012v5n4p188}

Carbajal, J. y D. Saur (coords.) (2010), El desafío tecnológico. Transformaciones y fronteras educativas, México, SADENE/Juan Pablos.

Carr, W. (1999), ¿Teoría, tecnología o praxis? El futuro de la educación del maestro. En Tlaseca

P., M. (coord.), El saber de los maestros en la formación docente, México, UPN, pp. 183-194.

Casas A., M. (1998), Tendencias actuales e innovaciones en la educación superior a distancia. Potencialidad y restricciones en Latinoamérica, Buenos Aires, Kapelusz

Castañeda, A. et al (2004), Formación, distancias y subjetividades. Nuevos retos de la formación en la globalización, México, UAEM/Limusa.

Cebrián M. y M. Gallego (2011), Procesos educativos con TIC en la sociedad del conocimiento, Madrid, Ediciones Pirámide.

Castells, M. (2008). La era de la información. Economía, sociedad y cultura, vol. 1, La sociedad Red, Madrid, Alianza Editorial.

Delors, J. (1997), La educación encierra un tesoro, México, UNESCO.

Drucker, Peter, (1994,) The Age of Social Transformation, en The Atlantic Monthly, Volume 273, Number 11, Boston.

Ferry, G. (1990) El trayecto de la formación. Los enseñantes entre la teoría y la práctica. México, Paidós.

García A., L. (2001), La educación a distancia. De la teoría a la práctica, Barcelona, Ariel Educación.

González, Á. (2008), Relación entre formación y tecnologías en la sociedad de la información. En: Martínez, F. y Prendes, M. (coords.). Nuevas tecnologías y Educación, España, Pearson Editores.

Gros Salvat, B. y J. Silva Quiroz (2005), La formación del profesorado docente en los espacios virtuales de aprendizaje, en Revista OEI, Universidad de Barcelona, España y Universidad de Santiago, Chile, Fecha de consulta 08/ 22/09, http://rusc.uoc.edu/rusc/5/I/dt/eng/benyousef_ragni.pdf

Instituto Latinoamericano de la Comunicación Educativa (2003), El uso de la tecnología en el aula. Programa Nacional para la Actualización Permanente de Maestros de Educación Básica en Servicio, México, ILCE.

Imbernón, F. (1998), La formación y el desarrollo profesional del profesorado. Hacia una nueva cultura profesional, Barcelona, Graó. 


\section{INVESTIGACIÓN COMPARADA EN EDUCACIÓN: PROCESOS DE INCORPORACIÓN DE LAS \\ TIC EN LA PRÁCTICA DOCENTE DEL SUAYED-FFYL UNAM \\ DOI: http://dx.doi.org/10.5007/1983-4535.2012v5n4p188}

McLuhan, Marshall (1989), The Global Village (with Bruce R. Powers), United Kingdom, Oxford University Press.

McLuhan, Marshall (1996), Comprender los medios de comunicación, Madrid, Paidós.

Robalino, M. y A. Korner (coords.) (2005), Formación docente y Tecnologías de la Información y Comunicación, París, UNESCO.

Rojas M., I. (1998), “Tendencias en la formación profesional del licenciado en Pedagogía. El caso de la carrera de Pedagogía del SUA de la UNAM”, tesis de maestría, México, UNAM-FFyL.

Rojas M., I. (2010), 1. La tutoría en el Sistema Universidad Abierta de la Facultad de Filosofía y Letras: definición y construcción de una modalidad de docencia en sistema abierto. En Ducoing, P. (coord.), Tutoría y mediación II, UNAM-IISUE-AFIRSE, pp. $27-57$.

Rojas M., I. (2011), Construcción de un esquema para la evaluación de docentes del Sistema Universidad Abierta y Educación a Distancia de la UNAM (en prensa), Bogotá, RIIED/UniAndes.

Rojas M., I. (2012), Procesos de formación, Estado del conocimiento 2002-2012. Informe de avances de la sub-área de formación y tecnologías (documento preliminar), México,

UNAM/COMIE.

Rojas M., I. et al. (1995), La asesoría y la evaluación en la formación docente en sistemas abiertos. En Revista Iberoamericana de Educación Superior a Distancia. Madrid: UNED/AIESAD. Vol. VII -N² 2, pp. 69-77.

Rojas M., I. et al (2001), Curso Formación y práctica docente de asesores en sistemas de educación abierta, México, UNAM-SUAFyL.

Rojas M., I y Z. Navarrete C. (2010), Educación comparada: reflexiones para la construcción de una metodología de investigación. En Navarro L., M. (Coord.) Educación Comparada. Perspectivas y casos, México, Planea/Sociedad Mexicana de Educación Comparada, pp. 53-66.

Rosalía, R. y Román, P. (2007), La formación del profesorado en las tecnologías de la información y de la comunicación. Las tecnologías en la formación del profesorado. En Cabero (coord.), Tecnología educativa, España, McGraw-Hill/Interamericana, pp. 141-158.

Rumble, G. (1998), La educación a distancia y la renovación necesaria de las universidades para el siglo XXI, Madrid, Morata.

Schriewer, J.(1993), El método comparativo y la necesidad de externalización: criterios metodológicos y conceptos sociológicos. En Schriewer, J. y F. Pedró (comps.), 
Manual de educación comparada (Vol. II) Teorías, investigaciones, perspectivas. Barcelona, Pomares, pp. 189-251.

Schriewer, J. (comp.) (2002), Formación del discurso en la educación comparada, Barcelona, Pomares.

Schriewer, J. et al (comps.) (2006), Redes intelectuales trasnacionales. Formas de conocimiento académico y búsqueda de identidades culturales, Barcelona, Pomares.

UNESCO (1990), Informe sobre la comunicación en el mundo, París.

UNESCO (1995). Documento de política para el cambio y desarrollo de la educación Superior, París; UNESCO.

UNESCO (1996). L’Unesco et une societé de l'information pour tous, París, UNESCO.

UNESCO (1997), Rapport mondial sur la communication. Les medias face aux défis des nouvelles technologies, París, UNESCO.

UNESCO (1998). La educación superior en el siglo XXI. Visión y acción. De lo tradicional a lo virtual: las nuevas tecnologías de la información, debate temático, París, UNESCO.

UNESCO (2004), Las tecnologías de la información y la comunicación en la formación docente. Guía de planificación, París, UNESCO.

UNESCO (2008), Estándares de competencia en TIC para docentes, París,

Vera C., M. (2005), "Formación docente de los tutores del Sistema Universidad Abierta de la Facultad de Filosofía y Letras (UNAM)", tesis de maestría, México, UNAM-FFyL.

Zbigniew, Brzezinski (1973), La era tecnotrónica, Buenos Aires, Paidós.3-66. 